\title{
The event rate context in vigilance: Relation to signal probability and expectancy
}

\author{
JUDITH E. KRULEWITZ \\ Iowa State University, Ames, Iowa 50010 \\ and \\ JOEL S. WARM \\ University of Cincinnati, Cincinnati, Ohio 45221
}

\begin{abstract}
The influence of the conditional probability of signals encountered during an adapting phase of a visual vigilance task on postadaptation performance was examined within the context of different background event rates (frequencies of presentation of repetitive neutral events). Slow ( 6 events $/ \mathrm{min}$ ) and fast ( 30 events $/ \mathrm{min}$ ) event rates were used. Within each event rate, four groups of subjects experienced either a low (.025) or a high (.125) conditional probability of signals for 20 min (adaptation phase) and then experienced either the same or the alternate probability for an additional $40 \mathrm{~min}$ (postadaptation phase). The overall frequency of correct detections was inversely related to event rate. With the fast event rate, subjects who were initially exposed to a high signal probability detected more signals during the postadaptation phase than those who received early exposure to a low signal probability. By contrast, the effects of signal probability were negligible within the context of a slow event rate. These results suggest some limits to the applicability of an expectancy theory of vigilance.
\end{abstract}

In most sustained attention or vigilance experiments, critical signals for detection occur in an infrequent and aperiodic manner. Consequently, observers are typically confronted with a good deal of temporal uncertainty as to when such signals will appear. The expectancy hypothesis (Baker, 1963) focuses upon the temporal uncertainty aspect of sustained attention tasks. According to this view, observers act as temporal averaging instruments and form expectancies or predictions about future signal appearances on the basis of a sampling of the event structure. Readiness to detect a signal is assumed to be positively related to the observer's level of expectancy.

Support for the expectancy notion comes from studies showing that the efficiency of vigilance performance is directly related to the conditional probability of critical signals and also to the temporal regularity with which they appear (Baker, 1963; Warm, 1977). Presumably, high signal probability and regular intervals of presentation enhance the observer's abilities to form accurate expectancies. According to Broadbent (1971), key evidence favoring the expectancy hypothesis has been provided in experiments by Colquhoun and Baddeley $(1964$, 1967) which demonstrate the perseverative effects of exposure to one level of signal

The authors are indebted to W. N. Dember, D. A. Schumsky, and R. G. Wait for their helpful comments and to O.W. Hall for technical assistance. Requests for reprints should be sent to Judith E. Krulewitz, Department of Psychology, Iowa State University, Ames, lowa 50010. probability on subsequent performance under a different level of probability. In these experiments, observers who were exposed to a high probability of signal appearances during an adapting phase of a vigilance session detected more signals in a subsequent phase than those who initially experienced a low probability. Similar findings have also been reported by McFarland and Halcomb (1970).

While evidence is available to demonstrate that expectancy is involved in vigilance performance, the limits within which expectancy determines performance have not been fully explored. One factor which seems to be important in this regard is the matrix of regularly occurring neutral events in which critical signals are embedded-the background event rate. Increments in event rate lower signal detectability (Davies \& Tune, 1969; Warm, 1977). They also modify the effects associated with the temporal structure of critical signal appearances. For example, Moore and Gross (1973) have reported that the enhancement of performance associated with regular critical signal presentations tends to show up earlier in the vigil when the rate of repetition of neutral events is fast rather than slow.

It is worth noting that the experiments by Colquhoun and Baddeley $(1964,1967)$ and McFarland and Halcomb (1970) on the perseverative effects of critical signal probability were conducted with an event rate of 30 events/min and that the generality of these effects to performance under different event rates is unknown. Accordingly, this investigation was designed to determine if the perseverative effects of critical signal 
probability are also dependent upon the event rate context in which critical signals appear.

\section{METHOD}

\section{Subjects}

One hundred and forty-four undergraduates from the University of Cincinnati, 72 women and an equal number of men, were the subjects. None had served previously in a vigilance experiment. The students took part to fulfill a course requirement.

\section{Design}

All subjects participated in a 1-h session divided into three 20 -min periods of watch. Two event rates were used; a slow rate of 6 events $/ \mathrm{min}$ and a fast rate of 30 events $/ \mathrm{min}$. Within each event rate, the subjects experienced either a low $(.025)$ or a high (.125) conditional probability of critical signals during the initial period of watch (adaptation phase) and then experienced either the same or the alternate conditional probability for the remainder of the session (postadaptation phase). Thus, within each event rate, there were four conditions of critical signal probability, high-high (HH), high-low (HL), low-low (LL), and low-high (LH). Eighteen subjects were assigned at random to each of these conditions with the restriction that gender be equated across groups.

\section{Apparatus}

The subjects monitored a visual display consisting of a $23 \times 17 \mathrm{~mm}$ rectangular light field surrounded by a thin white border, $2 \mathrm{~mm}$ in width, centered within an $89 \times 150 \mathrm{~cm}$ flatblack panel. The light field was illuminated by a 5-W NE ${ }_{42}$ neon bulb, operated from $117 \mathrm{~V}, 60 \mathrm{~Hz}$ ac, mounted behind a sheet of milk glass $(.64 \mathrm{~cm}$ thick) and a $20 \%$ neutral density filter. A stimulus event was a 1-sec light pulse. Slow and fast event rates of 6 and 30 events/min were produced by setting the interevent intervals at 9.0 and $1.0 \mathrm{sec}$, respectively. A noncritical or neutral event requiring no overt response was a light pulse with an intensity produced by $1.0452 \mathrm{~W}$ power. Critical signals for detection were light pulses with an intensity determined by $1.7621 \mathrm{~W}$ power. With a two-alternative forcedchoice psychophy sical procedure, critical signals were essentially always detected by alerted subjects under both the slow and the fast event rates.

At the slow event rate, either 3 or 15 signals, yielding conditional probabilities of .025 or .125 , respectively, were presented during each $20-\mathrm{min}$ period of watch. At the fast event rate, 15 or 75 critical signals, corresponding to conditional probabilities of .025 or .125 , respectively, were presented during each period. To control for relative variability, signals were chosen from similar positively skewed distributions of intersignal intervals. The sequence of intersignal intervals was varied at random for each subject during each 20-min period.

Stimulus event rates and the occurrence of critical signals were controlled by solid-state programming equipment and a punched-tape timer. The subjects responded to critical signals by depressing a hand-held pushbutton switch, and their responses were recorded on two ITT electronic counters. Responses occurring within a 2.0 -sec period after critical signal onset were automatically recorded as correct detections; all others were considered as false alarms. The 2.0-sec cutoff was based upon pilot work which indicated that if a subject is going to respond to a critical signal, he or she will do so within this time limit.

\section{Procedure}

Each subject was tested individually in a $1.5 \times 1.5 \times 2.1 \mathrm{~m}$ acoustically treated experimental chamber. The walls of the chamber were painted flat white, and ambient illumination of $4.0 \mathrm{fL}$ was provided by a light source mounted in a cylindrical ceiling fixture. The monitored display was mounted at eye level in a wall of the chamber. Subjects were seated without restraint and viewed the display from a distance of $86 \mathrm{~cm}$. All subjects wore Soundbarrier earmuffs, which, together with the noise of an exhaust fan, served to attenuate laboratory sounds. Control equipment and the experimenter were located outside the chamber. An intercom permitted voice communication as well as acoustic surveillance of the subject's activities during the session. The vigil proceeded from adaptation to postadaptation phases without interruption and, except for alterations in the conditional probability of critical signals, there was no discriminable change between periods. Subjects in the conditions in which signal probabilities were altered were not instructed as to the possibility of such alterations and did not anticipate them. All subjects surrendered their watches at the start of the session and had no knowledge of the length of the vigil other than that it would not exceed $2 \mathrm{~h}$.

\section{RESULTS}

The percentage of correct responses was calculated from the data of each subject for each 20-min period of watch. Mean percentages of correct detections are presented in Figure 1. Data for the slow and fast event

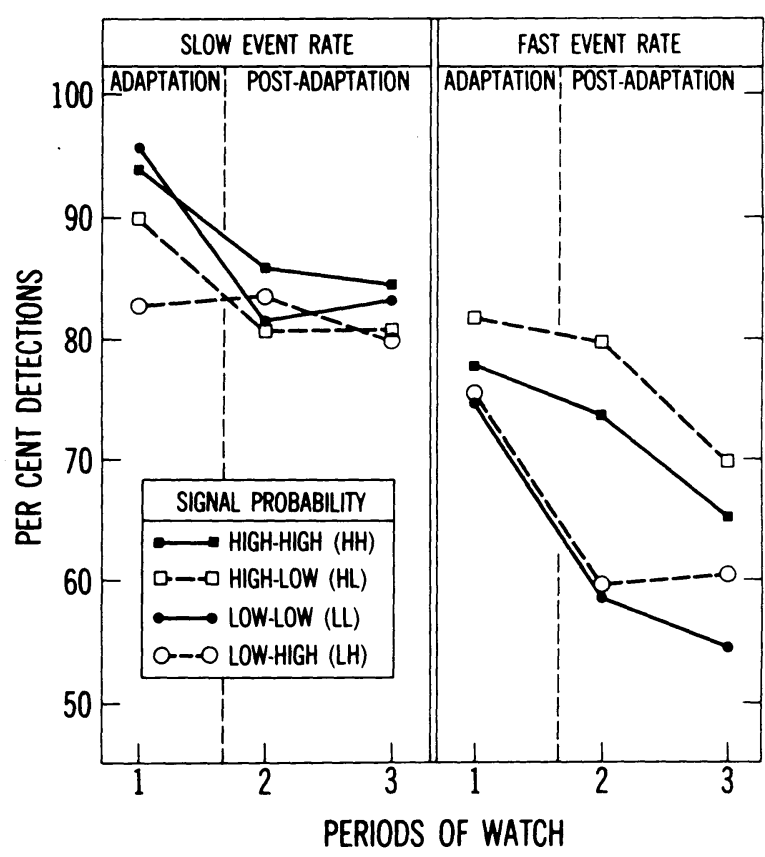

Figure 1. Percentage of correct responses as a function of periods of watch $(20 \mathrm{~min})$ for high and low signal probabilities during the adaptation and postadaptation phases of the study. Groups maintained on a constant level of signal probability are indicated by filled figures and solid lines; those which experienced a change in probability are indicated by open figures and dashed lines. Data for the slow (6 events $/ \mathrm{min}$ ) and the fast (30 events/min) event rates are presented separately in each panel. 
rates are presented separately in each panel. Within each event rate, the scores for the different conditions of signal probability are plotted as a function of periods of watch during the adaptation and postadaptation phases of the study.

\section{Adaptation Phase}

The data of the adaptation phase were examined for the effects of event rate and signal probability. In addition, the equivalence of the scores for the two groups having different potentials for shifts in probability within the factorial combinations of event rate and adapting signal probability was also assessed. An analysis of variance based on an arc-sine transformation of the data indicated that detection scores were significantly higher with the slow relative to the fast event rate $[F(1,136)=40.12, p<.001]$. None of the other main effects reached significance $(p>.05)$. Figure 1 suggests that when a fast event rate was used, the two levels of adapting signal probability may have yielded different detection frequencies. However, the Event Rate by Probability interaction, as well as all of the other interactions in this analysis, lacked significance $(p>.05)$.

\section{Postadaptation Phase}

The perseverative effects of the adapting signal probability were probed by comparing the scores for the shifted and nonshifted groups at each combination of postadaptation signal probability and period of watch. Figure 1 shows that within the context of a slow event rate, there were no systematic differences in postadaptation performance. By contrast, within the context of a fast event rate, systematic differences seem to have emerged among the scores for the various conditions of postadaptation probability, probability shift, and time on watch.

These impressions were confirmed by separate 2 (postadaptation probability) by 2 (probability shift) by 2 (periods of watch) analyses of variance on arc-sine transformations of the data for the slow and fast event rates. None of the main effects or interactions was significant at the slow event rate $(\mathrm{p}>.05$ in all cases). At the fast event rate, however, detections declined significantly over the $40 \mathrm{~min}$ of the postadaptation phase $[F(1,68)=9.40, p<.005]$. The overall effects of postadaptation probability and probability shift were not significant $(p>.05)$, but there was a significant interaction between these factors $[F(1,68)=8.62$, $\mathrm{p}<.005]$, as well as a significant double-order interaction between postadaptation probability, probability shift, and time $[F(1,68)=5.07, p<.05]$. All of the remaining interactions in the analysis lacked significance $(\mathrm{F}<1)$.

The significant interactions are evident in Figure 1. When the postadaptation signal probability was low, detection scores were greater for the group which experienced a probability shift (HL) than for the group which did not (LL). When the postadaptation probability was high, detection scores were poorer for the shifted group (LH) relative to the nonshifted group $(\mathrm{HH})$. In essence, postadaptation performance under the fast event rate was dominated by the signal probability experienced by the subjects during the adaptation phase. At both levels of postadaptation probability, detection scores were greater for those subjects who were initially exposed to a high as compared to a low level of signal probability. Figure 1 also reveals that the perseverative effects of the adapting signal probability were most pronounced during the first portion of the postadaptation phase.

\section{DISCUSSION}

On the basis of this study, we conclude that the perseverative effects of critical signal probability are indeed dependent upon the event rate context in which signals appear. With a rate of 30 events/min, the same as that used by Colquhoun and Baddeley $(1964,1967)$ and McFarland and Halcomb (1970), the adapting conditions influenced the postadaptation results. As in the earlier experiments with this event rate, prior exposure to a high signal probability produced better detection scores during the postadaptation phase than did prior exposure to a low signal probability. On the other hand, when the event rate was lowered to 6 events $/ \mathrm{min}$, the effects of signal probability were negligible.

As noted previously, the perseverative effects of critical signal probability have been considered as important evidence in favor of an expectancy approach to vigilance. The present results are not inconsistent with the implications of expectancy theory. They do, however, suggest some limits within which observers may effectively resort to an expectancy strategy in coping with the demands of a sustained attention task.

There are at least two factors which could have negated any effects of signal probability at the slow event rate in this study: (1) Accurate expectancies were precluded in this context or, (2) an expectancy strategy was not necessary to cope with task demands under the slow event rate. Recall that the critical signal densities differed under the two event rates. In the case of the slow event rate, the absolute number of critical signals was 3 per $20 \mathrm{~min}$ in the low-probability condition and 15 per $20 \mathrm{~min}$ in the high-probability condition. The comparable numbers of critical signals under the fast event rate were 15 per $20 \mathrm{~min}$ and 75 per $20 \mathrm{~min}$, respectively. Thus, it could be argued that the number of signals embedded in the slow event rate was too small to permit the generation of meaningful expectations by the observers. While it is true that the signal densities differed under the two event rates, the argument that these differences can account for the present results seems unlikely. Such an argument would imply that detection scores for the slow event rate should be poorer than those for the fast event rate, an implication which is just the opposite of the result which actually occurred.

At this point, we favor an interpretation in which the slow event rate is viewed as a context which did not foster reliance on an expectancy strategy. Specifically, Baker (1963) has noted that precise expectancies are not formulated easily and that they require some effort on the part of the observer. In addition, Jerison $(1970,1977)$ has suggested that increments in event rate increase the pace at which observers must attend to the vigilance display and that this brings with it an accumulation of inhibitory-like processes which make attending increasingly more difficult. Therefore, it is conceivable that under the slow 
event rate, task demands were slight and the observers were able to detect critical signals without the need to resort to expectancy information. Under the fast event rate, however, an expectancy strategy could have been more useful. Such a strategy could serve to reduce increased task demands by restricting the observer's attention to those intervals when a signal was considered "most likely" to appear on the monitored display. An interpretation along these lines follows the Davies and Tune (1969) proposal that dependence upon expectancy in vigilance is an information-processing strategy which may be apparent primarily when task demánds are heavy and easier options are not available to the observer.

\section{REFERENCES}

BAKER, C. H. Further toward a theory of vigilance. In D. $\mathrm{H}$. Buckner \& J. J. McGrath (Eds.), Vigilance: A symposium. New York: McGraw-Hill, 1963.

Broadbent. D. E. Decision and stress. New York: Academic Press, 1971.

Colquhoun, W. P.. \& Baddeley, A. D. Role of pretest expectancy in vigilance decrement. Journal of Experimental Psvchology, 1964, 68, 156-160.

Colquhoun, W. P., \& Baddeley, A. D. Intluence of signal probability during pretraining on vigilance decrement. Journal of Experimental Psychology, 1967, 73. 153-155.

Davies. D. R., \& TUNE, G. S. Human vigilance performance. New York: American Elsevier. 1969.
JERISON, H. J. Vigilance, discrimination and attention. In D. I. Mostofsky (Ed.). Attention: A behavioral analysis. New York: Appleton-Century-Crofts. 1970.

JERISON, H. J. Vigilance: Biology, psychology, theory and practice. In R. R. Mackie (Ed.), Vigilance: Theory, operational performance and physiological correlates. New York: Plenum. 1977.

McFarland, B. P., \& Halcomb, C. G. Expectancy and stimulus generalization in vigilance. Perceptual and Motor Skills, 1970, 30, 147-151.

Moore, S. F., \& Gross, S. J. Influence of critical signal regularity, stimulus event matrix, and cognitive style on vigilance performance. Joumal of Experimental Psychology. 1973, 99. 137-139.

WArm. J. S. Psychological processes in sustained attention. In R. R. Mackie (Ed.), Vigilance: Theory, operational performance and physiological correlates. New York: Plenum. 1977.

\section{NOTE}

1. In the earlier studies by Colquhoun and Baddeley (1964, 1967), signal detection measures, $d^{\prime}$ and $\beta$, were used to probe the perseverative effects of signal probability. These measures are not presented in this report because low false alarm rates precluded their meaningful application to the data.

(Received for publication August 11, 1977.) 